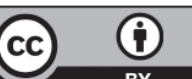

https://creativecommons.org/licenses/by/4.0/

\title{
SELECCIÓN Y ANÁLISIS DE ECUACIONES ANTROPOMÉTRICAS PARA EL CÁLCULO DE LA COMPOSICIÓN CORPORAL EN ADULTOS
}

\author{
Selection and analysis of anthropometric equations \\ for the calculation of body composition in adults
}

ALDO PIÑEDA GERALDO*, YULIANA GONZÁLEZ RINCÓN**,

Recibido: 21 de octubre de 2016. Aceptado: 21 de noviembre de 2016

DOI: http://dx.doi.org/10.21017/rimci.2017.v4.n7.a21

\begin{abstract}
Resumen
En diversos estudios se han utilizado criterios antropométricos, como el índice de masa corporal (peso-estatura), para definir la obesidad, no obstante estos índices poseen poca sensibilidad para monitorear y calcular realmente el sobrepeso y la obesidad. Por lo anterior y en la búsqueda de mejores opciones, se evaluaron ecuaciones antropométricas de la composición corporal para poder identificar las de mayor sensibilidad para calcular las proporciones corporales. El presente trabajo tuvo como objetivo seleccionar y analizar ecuaciones antropométricas para el cálculo de la composición corporal. El estudio fue descriptivo, se evaluaron dos individuos masculino y femenino para verificar la utilidad de las ecuaciones elegidas. Para la recopilación de los datos se diseñó una ficha antropométrica, las medidas más sobresalientes estudiadas fueron los pliegues cutáneos: tricipital, subescapular, suprailíaco, abdominal, muslo anterior y pierna (pantorrilla). Dos diámetros: el biestiloideo (muñeca) y el bicondileo (femoral) y se analizaron las ecuaciones de Yuhasz, el de Rocha, Matiegka y la ecuación de Dubois-Dubois.
\end{abstract}

Palabras clave: selección, análisis, ecuaciones antropométricas, cálculo, composición corporal.

\begin{abstract}
Several studies have used anthropometric criteria, such as body mass index (weight-height), to define obesity, but these indexes have little sensitivity to actually monitor and calculate overweight and obesity. For the above and in the search for better options, anthropometric equations of body composition were evaluated in order to identify those with greater sensitivity to calculate body proportions. The present work aimed to select and analyze anthropometric equations for the calculation of body composition. The study was descriptive, we evaluated two male and female individuals to verify the utility of the chosen equations. For data collection, an anthropometric record was designed. The most important measures were the skin folds: triceps, subscapular, suprailiac, abdominal, thigh and calf. Two diameters: the biestiloideo (wrist) and the bicondileo (femoral) and analyzed the equations of Yuhasz, that of Rocha, Matiegka and the equation of Dubois-Dubois.
\end{abstract}

Keywords: selection, analysis, anthropometric equations, calculation, body composition.

\footnotetext{
* Antropólogo Físico de la Escuela Nacional de Antropología e Historia de México, D.F. Posgrado en Ergonomía de la Universidad El Bosque. Docente-Investigador de la Facultad de Ingeniería Industrial de la Corporación Universitaria Republicana, Bogotá. Colombia. Correo electrónico: apinedaergocolmex@gmail.com

** Estudiante del semillero del grupo OCA. Perteneciente a la Facultad de Ingeniería Industrial. Correo electrónico: ing.industrial04@gmail.com

*** Estudiante del semillero del grupo OCA. Perteneciente a la Facultad de Ingeniería Industrial. Correo electrónico: sapadial17@gmail.com

***** Estudiante del semillero del grupo OCA. Perteneciente a la Facultad de Ingeniería Industrial.
} 


\section{INTRODUCCIÓN}

$E_{\text {ste proyecto de investigación aplicada se de- }}$ sarrolló con el grupo de investigación OCA (Operaciones, Calidad y Administración) de la Corporación Universitaria Republicana, en el que se incorporan estudiantes de la facultad de ingeniería Industrial. En el presente estudio el objetivo fue seleccionar y analizar ecuaciones antropométricas para calcular la composición corporal en adultos. Para el logro de las ecuaciones antropométricas se seleccionan las principales ecuaciones antropométricas relacionadas con la composición corporal, así mismo, se realizaron pruebas de las ecuaciones antropométricas para verificar su utilidad.

La composición corporal es aquella rama de la biología humana que se ocupa de la cuantificación en vivo de los componentes corporales, las relaciones cuantitativas entre los componentes y los cambios cuantitativos en los mismos relacionados con varios factores influyentes [1]. Por otro lado, se puede definir como el estudio de la composición corporal como la evaluación por distintos métodos, de las diferentes fracciones corporales consideradas, respecto del peso total [2]. Así mismo, la cuantificación de los componentes corporales es fundamental para estudios de obesidad. En este sentido, los estudios de composición corporal se centran en tres áreas interconectadas: primero los componentes y sus interrelaciones, segundo el desarrollo y la evaluación de sus métodos para su medición y los factores que influyen [3]. En este sentido podemos diferenciar tres áreas para el estudio de la composición corporal que son: determinación de los componentes principales (cuantificación y distribución), técnicas de medición (directas e indirectas) y los análisis de los factores que influyen sobre la composición corporal.

El cuerpo humano, está formado por 11 elementos que son responsables de más del $99 \%$ de su peso total. La composición corporal se puede estudiar a nivel atómico, a nivel molecular (sus componentes son: el agua, los lípidos, proteínas, minerales y carbohidratos). A nivel celular (está compuesto por grasa, masa celular corporal, líquido extracelular y sólidos extracelulares). A nivel de tejidos y sistemas (sus principales componentes son: tejido adiposo, el musculo esquelético, los huesos y los órganos). Y a nivel del cuerpo total, (se estudia bajo: la masa, la densidad corporal, estatura, resistencias, perímetros, pliegues cutáneos, anchuras, longitudes) [3].

Estos niveles están relacionados entre sí, lo que quiere decir es que un nivel puede ser trasladado a otro nivel. Aspecto de interés para el presente trabajo, ya que constituye la base de numerosas técnicas para evaluar, determinar y analizar la composición corporal. A continuación, se va a mencionar algunas técnicas para determinar y calcular la composición corporal en términos de métodos directos, indirectos y doblemente indirectos.

En los métodos directos los valores son precisos, pero son técnicas invasivas, por lo que la hacen menos accesibles. Estos, nos dan valores directos sin necesidad de transformaciones posteriores. De estos métodos sobresalen: la disección de cadáveres y la biopsia de tejidos [4].

Entre las técnicas indirectas, están el pesaje hidrostático, plestimografía, técnicas isotópicas: agua corporal total, dilución isotópica. Potasio corporal total: espectrometría. Técnicas químicas: determinación de creatinina. Técnicas de imagen: resonancia magnética nuclear (RMN), tomografía computarizada (TC), absorciometría fotónica dual de rayos $X$ (DEXA) [4].

Las técnicas doblemente indirectas, son técnicas de campo que son validadas a través de métodos indirectos. Entre ellos están: las técnicas antropométricas, bioimpedancia eléctrica, conductividad eléctrica corporal total (TOBEC), técnica de imagen por ultrasonidos [4]. El elevado costo y difícil uso de estas técnicas hacen que no sean utilizadas frecuentemente, excepto la impedancia bioeléctrica y la antropometría, que por su sencillez, bajo costo, rapidez, grado de confiabilidad, son los que se van a elegir para su aplicación. Adicionalmente, el carácter no invasivo de las mediciones antropométricas y de bioimpedancia favorece su aplicación [4].

Los estudios antropométricos permiten calcular la composición corporal, el estudio de la morfología, las dimensiones y la proporcionalidad en relación a la ergonomía. Se tiene una variedad de métodos y técnicas para valorar la composición corporal, que han sido analizadas y desarrolladas. Entre las cuales se va a destacar la antropometría

Rev. Ingeniería, Matemáticas y Ciencias de la Información Vol. 4 / Núm. 7 / enero-junio de 2017; pág. 47-56 
como una técnica de fácil aplicación, buena reproductibilidad y bajo costo. Este método, es el más recomendable ya que es sencillo, reproducible, accesible, cómodo y económico. La confiabilidad dependerá de la habilidad y la experiencia del antropometrista en la toma de las medidas y la calibración del mismo. El protocolo deberá ser estandarizado de acuerdo a las normas internacionales, para que puedan ser comparables los resultados entre los diferentes grupos de trabajadores en los sectores productivos.

La propuesta de este proyecto es justamente dar a conocer la importancia que tiene desde el enfoque ergonómico estas técnicas. La antropometría es una herramienta de la ergonomía, ya que describe, analiza, calcula y evalúa los diferentes factores y características físicas de los trabajadores en relación con sus espacios laborales. Además, valora la parte funcional del trabajador(a), donde se incluye el estudio del perfil antropométrico y corporal, por ser uno de los tantos factores que influyen en la actividad laboral, tanto desde el aspecto fisiológico, el esfuerzo, levantamiento de carga, fatiga y desde el aspecto biomecánico.

Los compartimientos corporales, pueden estudiarse como modelos multicompartimentales o simplificarse en modelos más sencillos, tetra, tri, bi o monocompartimentales. Esta diferenciación compartimental que con mayor frecuencia se suele hacer en el cuerpo es: la fracción grasa (masa grasa) y fracción magra (masa libre de grasa) [4].

La siguiente técnica es beneficiosa y será objeto de nuestro estudio, que consiste en valorar métodos doblemente indirectos que dependen de una relación estadística entre parámetros corporales fácilmente medibles y el componente corporal de interés [5]. Estos datos antropométricos son procesados mediante diferentes ecuaciones de regresión y formulas estadísticas para obtener información sobre la composición corporal. Estos datos van a aportar información sobre los componentes del cuerpo humano en los trabajadores, para conocer los porcentajes de grasa, masa muscular, masa ósea y residual. Uno de los objetivos del proyecto es hacer una recolección de ecuaciones, formulas e índices antropométricos que puedan ser aplicables a la población trabajadora colombiana.
Esta técnica es basada en medidas antropométricas que a continuación relacionamos, para los cálculos de composición corporal: Complexión corporal [6].

El índice peso/estatura (IMC): Donde se establece la relación del peso con el cuadrado de la estatura, para compensar la no linealidad del peso en la siguiente ecuación [7]:

$$
\mathrm{IMC}=\frac{\text { peso }}{\text { estatura }^{2}}
$$

Donde el peso se expresa en kilogramos (Kg) y el cuadrado de la estatura en metros cuadra$\operatorname{dos}(\mathrm{m})^{2}$.

Análisis de la composición corporal, índices corporales porcentaje de grasa corporal: es la fracción relativa de la masa grasa corporal total. Se accede a su cálculo a través del modelo fundamental de la composición corporal, mediante los diferentes procedimientos para su evaluación. De acuerdo a la ecuación de Deurenberg permite el cálculo del porcentaje de grasa corporal.

$$
\begin{aligned}
& (1,12 \times \text { IMC }+(0,23 \times \text { edad en años })- \\
& (10,8 \times \text { Sexo })-5,4)
\end{aligned}
$$

donde se coloca el valor 0 para el sexo femenino y el valor 1 para el sexo masculino [8].

Medición de la grasa corporal mediante impedancia bioléctrica, pliegues cutáneos y ecuaciones a partir de medidas antropométricas [8].

De acuerdo a Yuhasz, el porcentaje de masa grasa se puede calcular en hombres y mujeres con las siguientes fórmulas:

$$
\begin{aligned}
& \text { \%M.G. }(\text { Fem })=4,56+(\text { S } 6 \text { pliegues }(\mathrm{mm}) \times 0,143) \\
& \text { \%M.G. }(\text { Masc })=3,64+(\text { S } 6 \text { pliegues }(\mathrm{mm}) \times 0,097)
\end{aligned}
$$

donde los pliegues son: Tríceps, subescapular, suprailíaco $(2 \mathrm{~cm}$ por delante de la línea axilar media), abdominal, muslo anterior y pierna [9].

De acuerdo a Faulkner en 1980, establece el siguiente modelo de ecuación a partir de cuatro componentes para obtener el porcentaje de masa grasa: 
$\%$ M.G. $($ Masc $)=($ S 4 pliegues $\times 0,153)+5,783$

$\%$ M.G. $($ Fem $)=(S 4$ pliegues $\times 0,213)+7,9$

En la que los pliegues son: Tricipital, subescapular, suprailíaco y abdominal [9].

Masa grasa: representa la reserva energética corporal, que acompaña a la actividad metabólica de la masa magra. La función es energética y sirve de sostén y como un aislante térmico. Su densidad es de $0.9 \times 10 \mathrm{hg} / \mathrm{m}$ y se caracteriza por ser relativamente constante. La cantidad normal para hombres es de $15 \%$ y para mujeres $22 \%$. Para obsesos en hombres más del $25 \%$ y para mujeres obesas con más del $33 \%$ [10].

Peso óseo: La masa ósea es uno de los componentes que influye sobre el peso corporal, que varía en los diferentes grupos humanos [11]. De acuerdo a Rocha que dio lugar al desarrollo del modelo de la ecuación para calcular el peso óseo en la siguiente fórmula:

$$
\begin{aligned}
\text { Masa ósea }(\mathrm{Kg})= & 3,02 \times\left(\text { estatura }^{2} \times\right. \\
& \text { DM } \times \text { DF } \times 400)^{0,712}
\end{aligned}
$$

donde la estatura es en metros; DM es el diámetro de la muñeca en metros y DF es el diámetro del fémur en metros [12][13].

Otros autores como Rosen y Guimaraes, proponen otra ecuación para calcular la masa ósea que es igual a:

$$
3,02 \times\left(\frac{\text { estatura }^{2}}{100} \times \frac{\text { DM }}{100} \times \frac{\text { DF }}{100} \times 400\right) 0,712
$$

donde la estatura es en metros; DM es el diámetro de la muñeca en metros y DF es el diámetro del fémur en metros [14][15].

Superficie corporal: Con el peso y la estatura se puede calcular la superficie corporal, existen 11 fórmulas que a continuación se mencionará: Mosteller, 1987. Haycock, 1978. Biering, 1934. Dubois-Dubois, 1916. Boyd, 1939. Gehan, 1970. Isackson, 1936. Breitman, 1932. Von Schelling, 1954. Vierordt, 1906 y Bardeen 1920.

Dubois-Dubois en 1916, estableció la siguiente ecuación para calcular la superficie corporal [15].

$$
\mathrm{SC}=0,007184 \times \text { Peso }(\mathrm{Kg})^{0,425} \times \text { estatura }(\mathrm{cm})^{0.725}
$$

Mosteller en 1987, estableció la siguiente ecuación para calcular la superficie corporal [15]:

$$
\mathrm{SC}=(\text { Peso }(\mathrm{Kg}) \times \text { estatura }(\mathrm{cm}) / 3600)^{0,5}
$$

Superficie corporal como indicador de masa muscular en el adulto del sexo masculino.

Peso residual, la masa residual se halla mediante las constantes propuestas por Wurch en 1974.

$$
\begin{aligned}
& \text { masa residual }=(K g) \text { Ptx 24,1/100 (hombres) } \\
& \text { masa residual }=(K g) \text { Ptx 20,9 / } 100 \text { (mujeres) }
\end{aligned}
$$

donde; Pt es igual al peso corporal [16].

Masa muscular: La cuantificación de la masa muscular, ha mostrado el interés por conocer los aspectos de la composición corporal y su relación con el estado de las reservas proteicas, la capacidad termorreguladora y la independencia funcional [15].

De acuerdo con Martín 1964 la fórmula usada para medir la Masa Muscular es:

$$
\begin{aligned}
& \mathrm{mm}(\text { mujeres })= 32,71 \mathrm{Ga}^{2}+4,155 \mathrm{Gd}^{2}+4,090 \mathrm{Gc}^{2}- \\
& 2149(\mathrm{r} 0,966 ; \mathrm{SE}=1427 \mathrm{~g})
\end{aligned}
$$

$\mathrm{mm}$ (varones) $=39,31 \mathrm{Ga}^{2}+9,669 \mathrm{Gd}^{2}+10,48 \mathrm{Gc}^{2}-$

$$
7993(\mathrm{r} 1 ; \mathrm{SE}=408 \mathrm{~g})
$$

donde: MM masa muscular (gr), Ga: Perímetro antebrazo-pliegues cutáneos (cm), Gb: Perímetro pierna-pliegue cutáneo (cm), Gc: Perímetro Brazopliegues cutáneos (bíceps+tríceps), Gd: Perímetro muslo pliegue cutáneo [16].

El estudio de la composición corporal, posibilita el análisis de las variaciones en los componentes corporales asociados a los diferentes estudios como son: la temperatura corporal y los cambios originados por el balance energético y la actividad laboral. El conocimiento para la evaluación del estado nutricional. El aspecto físico de un trabajador(a), el equilibrio óptimo entre el peso corporal y la relación adecuada entre el peso graso y peso libre de grasa. El perfil de distribución de grasa y el desarrollo muscular, dependiendo de la actividad laboral. 
La antropometría es un medio para hacer estudios de composición corporal, por ser rápido, seguro y de bajo costo [7]. Mientras que algunos métodos son de alto costo, por los aparatos y requerimiento de personal especializados para el manejo de los mismos. Otros presentan riesgos de radiación por el tiempo necesario para la obtención de información.

Sin embargo, el uso de las variables antropométricas para valorar la composición corporal, tiene inconvenientes por lo que recomendamos tomar algunas medidas. La primera recomendación es seleccionar y aplicar ecuaciones que hayan sido validadas en la población que se va a estudiar, para reducir el error debido al efecto de las variaciones poblacionales, principio de la especificidad poblacional de las ecuaciones de estimación. En ese sentido, se recomienda aplicar ecuaciones específicas, si se realiza el estudio en poblaciones definidas como la población trabajadora colombiana. Para aplicarla se debe tener en cuenta la edad de los evaluados, la diversidad dentro del grupo específico, la diversidad de los grupos. Tratando de buscar semejanzas en las poblaciones, grupos de referencia estudiados [18].

El análisis de estas medidas antropométricas es una herramienta de gran utilidad para las áreas de ergonomía y salud ocupacional. La información y los resultados que arrojará el software de la composición corporal para trabajadores, será con aplicación ergonómica y suministrará también información acerca de estudios ergonómicos. Como se mencionó arriba, existen muchos métodos para recolectar información y datos de mediciones antropométricas, algunas tienen ventajas y otras limitaciones. Existen diferentes modelos, métodos y técnicas para evaluar la composición corporal. La elección de estos, depende del objetivo del estudio, del grado de precisión y exactitud que requiera la evaluación, los recursos y medios disponibles de instrumentos de medición.

\section{Metodología}

El tipo de estudio fue descriptivo, ya que se describieron las medias antropométricas para cada sujeto que se midió, el enfoque fue cuantitativo. Se realizó una primera evaluación y después de aproximadamente cinco se realizó una primera evaluación. A los sujetos, se les informó sobre que mediciones se tomarían para la realización y desarrollo del estudio se requirió de un espacio adecuado con privacidad, limpio, buena iluminación para la lectura de los instrumentos y de los datos.

Los puntos anatómicos de referencias se deben identificar con mucho cuidado y marcarse con un lápiz dermográfico, para posteriormente pasar a realizar las mediciones, tratando de efectuar una secuencia de arriba hacia abajo. El diseño para las tomas de las medidas fue el siguiente: todas las medidas se deben tomar del lado derecho y se realizan las medidas tres veces. Se evaluaron diez medias antropométricas siendo las siguientes: peso, estatura, seis pliegues subcutáneos y dos diámetros. El peso se tomó en kilogramos, la estatura en centímetros. Se evaluaron seis pliegues subcutáneos de acuerdo al siguiente orden: primero el pliegue tricipital, subescapular, suprailíaco, abdominal, muslo anterior y pierna (pantorrilla). Dos diámetros el de la muñeca y el femoral. Siguiendo el siguiente procedimiento, la persona debe estar de pie, deberá tener los brazos descansados. Posteriormente con un marcador rojo (lápiz rojo), se marcan el punto acromial siendo un punto de referencia.

El antebrazo se flexiona a $90^{\circ}$, posteriormente se marca el otro punto de referencia en el olecranon, después con una cinta métrica se miden los dos puntos y se dividen en dos y se marca en el antebrazo tricipital. Con el dedo índice pulgar se toma el pliegue en el punto de referencia (antebrazo), con el calibrador del panículo adiposo, se mide el pliegue tricipital.

El subescapular se mide en el ángulo inferior del subescapular. En el suprailíaco, se identificó con el índice pulgar el hueso iliaco, se identifica el punto de referencia y se agarra con el índice pulgar el pliegue y se realizó la medición. El abdomen con el lápiz rojo, se marcó el ombligo como punto de referencia, con el índice pulgar se toma el pliegue y se hace la medición. El muslo anterior, lo primero que se hizo fue identificar el punto medio de la pierna y con el índice pulgar se coge el pliegue para hacer la medida. Y se tomó el pliegue de la pantorrilla haciendo la identificación de la parte media, con el índice pulgar para hacer la medición. 
Por otro lado, se tomaron dos medidas más. El diámetro biestiloideo (muñeca), la técnica se hizo con el siguiente procedimiento: Se extendió el antebrazo a $90^{\circ}$ hacia arriba, se identifican los dos puntos óseos (biestiloideos) con los dos dedos índices, se marcan los dos puntos y con el calibrador de ramas cortas por ambas caras se abre y con las dos ramas se mide con el calibrador. Finalmente, el diámetro bicondileo (femoral), se identificaron con los dos índices los bicondileos, se marcaron los dos puntos con lápiz rojo, para posteriormente medir con el calibrador la anchura femoral.

La muestra fueron dos sujetos, una mujer y un hombre adulto. Los instrumentos fueron: Una ficha antropométrica que se diseño a propósito, ya que nos permitió recolectar y organizar los datos de cada sujeto evaluado (ver ficha). Los instrumentos de medición que se utilizaron fueron una balanza, una cinta métrica, un calibrador de panículo adiposo subcutáneo marca Whitehouse Skinfold Caliper, marca Holtain Tanner que fue diseñado en la Universidad de Londres y el Instituto de Salud de Niños. El rango del aparato es de 0 a $48 \mathrm{~mm}$. Tiene una precisión constante de $10 \mathrm{gr} / \mathrm{mm} 2$. Tiene un peso de $4 \mathrm{~kg}$. y una graduación de $0.2 \mathrm{~mm}$. La precisión es alta, sin embargo es necesaria una adecuada técnica de medición y un calibrador de ramas cortas. Ver tablas I y II.

\section{Ficha antropométrica. Composición corporal}

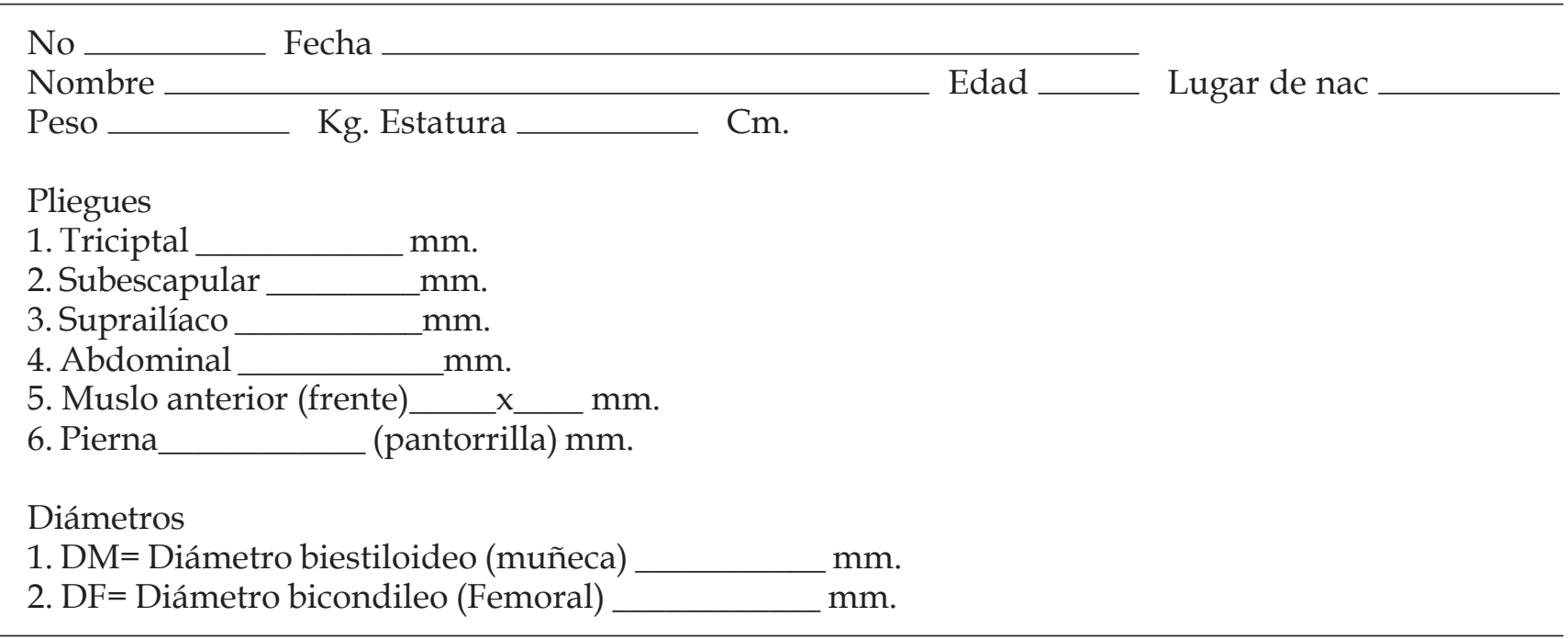

Tabla I. Ficha antropométrica. Composición Corporal - Modelo Femenino.

\begin{tabular}{|c|c|c|c|c|c|}
\hline $\begin{array}{l}\text { No } 1 \\
\text { NOMBRE: } \\
\text { PESO: } 42 \mathrm{Kg} .\end{array}$ & $\begin{array}{l}\text { FECHA: } \\
\text { Modelo: Femenino } \\
\text { ESTATURA: } 1.58 \mathrm{~m}\end{array}$ & & $: 24$ & LUGAR D & \\
\hline \multicolumn{2}{|l|}{ Pliegues } & Medida 1 & Medida 2 & Medida 3 & Promedio \\
\hline \multirow{6}{*}{\multicolumn{2}{|c|}{$\begin{array}{l}\text { 1. Tripcipital } \\
\text { 2. Subescapular } \\
\text { 3. Suprailíaco } \\
\text { 4. Abdominal } \\
\text { 5. Muslo anterior } \\
\text { 6. Pierna }\end{array}$}} & $14,6 \mathrm{~mm}$ & $14,2 \mathrm{~mm}$ & $14,2 \mathrm{~mm}$ & $14,3 \mathrm{~mm}$ \\
\hline & & $12 \mathrm{~mm}$ & $12,4 \mathrm{~mm}$ & $12 \mathrm{~mm}$ & $12,1 \mathrm{~mm}$ \\
\hline & & $10,6 \mathrm{~mm}$ & $11,4 \mathrm{~mm}$ & $11,4 \mathrm{~mm}$ & $11,1 \mathrm{~mm}$ \\
\hline & & $19 \mathrm{~mm}$ & $18,4 \mathrm{~mm}$ & $18 \mathrm{~mm}$ & $18,5 \mathrm{~mm}$ \\
\hline & & $14,2 \mathrm{~mm}$ & $14 \mathrm{~mm}$ & $12,6 \mathrm{~mm}$ & $13,6 \mathrm{~mm}$ \\
\hline & & $9 \mathrm{~mm}$ & $8,8 \mathrm{~mm}$ & $8,4 \mathrm{~mm}$ & $8,7 \mathrm{~mm}$ \\
\hline \multicolumn{2}{|l|}{ Diámetros } & Medida 1 & Medida 2 & Medida 3 & Promedio \\
\hline \multirow{2}{*}{\multicolumn{2}{|c|}{$\begin{array}{l}\text { 1. DM = Diámetro biestiloideo (muñeca) } \\
\text { 2. } \mathrm{DF}=\text { Diámetro bicondileo (femoral) }\end{array}$}} & $50,2 \mathrm{~mm}$ & $55,1 \mathrm{~mm}$ & $50,3 \mathrm{~mm}$ & $50,2 \mathrm{~mm}$ \\
\hline & & $80,3 \mathrm{~mm}$ & $80,2 \mathrm{~mm}$ & $79 \mathrm{~mm}$ & $79,8 \mathrm{~mm}$ \\
\hline
\end{tabular}


Tabla II. Ficha antropométrica. Composición Corporal - Modelo Masculino.

\begin{tabular}{|c|c|c|c|c|c|}
\hline \multirow{2}{*}{$\begin{array}{l}\text { No2 } \\
\text { NOMBRE: } \\
\text { PESO: } 76 \mathrm{Kg} \text {. } \\
\text { Pliegues }\end{array}$} & $\begin{array}{l}\text { FECHA: } 12 \text { de } \\
\text { Modelo: Mas } \\
\text { ESTATURA: } 1\end{array}$ & o de 2015 & $: 27$ & Modelo: Masculino & \\
\hline & & Medida 1 & Medida 2 & Medida 3 & Promedio \\
\hline 1. Tripcipital & & $8,4 \mathrm{~mm}$ & $8,2 \mathrm{~mm}$ & $8 \mathrm{~mm}$ & $8,2 \mathrm{~mm}$ \\
\hline 2. Subescapular & & $12,2 \mathrm{~mm}$ & $13 \mathrm{~mm}$ & $13 \mathrm{~mm}$ & $12,7 \mathrm{~mm}$ \\
\hline 3. Suprailíaco & & $22,8 \mathrm{~mm}$ & $23 \mathrm{~mm}$ & $22,8 \mathrm{~mm}$ & $22,9 \mathrm{~mm}$ \\
\hline 4. Abdominal & & $24 \mathrm{~mm}$ & $26 \mathrm{~mm}$ & $28 \mathrm{~mm}$ & $26 \mathrm{~mm}$ \\
\hline 5. Muslo anterior & & $14 \mathrm{~mm}$ & $14 \mathrm{~mm}$ & $14,2 \mathrm{~mm}$ & $14,1 \mathrm{~mm}$ \\
\hline 6. Pierna & & $11,6 \mathrm{~mm}$ & $11,4 \mathrm{~mm}$ & $11,4 \mathrm{~mm}$ & $11,5 \mathrm{~mm}$ \\
\hline Diámetros & & Medida 1 & Medida 2 & Medida 3 & Promedio \\
\hline 1. $\mathrm{DM}=$ Diámetro & ideo (muñeca) & $52 \mathrm{~mm}$ & $51 \mathrm{~mm}$ & $51 \mathrm{~mm}$ & $51,3 \mathrm{~mm}$ \\
\hline 2. $\mathrm{DF}=$ Diámetro & eo (femoral) & $96 \mathrm{~mm}$ & $98 \mathrm{~mm}$ & $96 \mathrm{~mm}$ & $96,7 \mathrm{~mm}$ \\
\hline
\end{tabular}

\section{Resultados}

Se seleccionaron siete ecuaciones antropométricas para calcular la composición corporal (ver Tabla III) que fueron las siguientes: el porcentaje de grasa, masa grasa, peso óseo, peso residual, masa muscular, índice de masa corporal y la superficie corporal. Los resultados de las ecuaciones seleccionadas (Ver Tabla IV) fueron las siguientes: la ecua- ción de Yuhasz, nos arrojó un porcentaje de grasa para la modelo femenina con un $15.8 \%$ de grasa. La masa grasa para la modelo femenino fue con un peso de $6.64 \mathrm{Kg}$. Y para el hombre de $9.81 \mathrm{~kg}$. El peso óseo para la mujer fue de $8.03 \mathrm{~kg}$. y para el hombre de $10 \mathrm{~kg}$. En cuanto a la masa muscular para el hombre fue de $38 \mathrm{~kg}$. y para la mujer fue de $18 \mathrm{~kg}$. Por su parte la masa residual para el modelo femenino es de $9 \mathrm{~kg}$ y para el hombre de $18 \mathrm{~kg}$.

Tabla III. Ecuaciones antropométricas.

\begin{tabular}{|c|c|c|}
\hline 1. & Porcentaje de Grasa Corporal & $\begin{array}{c}\text { Ecuación de Yuhasz } \\
\text { \%M. G. }(\text { Fem })=4,56+(\mathbf{\Sigma} \text { 6pliegues }(\mathbf{m m}) \times \mathbf{0}, \mathbf{1 4 3}) \\
\% \text { M. G. }(\text { Masc })=3,64+(\mathbf{\Sigma} \text { 6pliegues }(\mathbf{m m}) \times \mathbf{0}, \mathbf{0 9 7})\end{array}$ \\
\hline 2. & Masa Grasa & Peso graso $=\frac{\% \text { de grasa } \mathrm{x} \text { peso total }}{100}$ \\
\hline 3. & Peso Óseo & $\begin{array}{c}\text { Ecuación de Rocha } \\
\text { Masa ósea }(\mathrm{Kg})=3,02 \times\left(\text { estatura }^{2} \times \mathrm{DM} \times \mathrm{DF} \times 400\right)^{0,712}\end{array}$ \\
\hline 4. & Peso Residual & $\begin{array}{l}\text { Ecuación de Würch } \\
\text { Masa residual }=(\mathrm{Kg}) \text { Ptx24.1/100(hombres) } \\
\text { Masa residual }=(\mathrm{Kg}) \text { Ptx20.9/ 100(mujeres })\end{array}$ \\
\hline 5. & Masa Muscular & $\begin{array}{c}\text { Ecuación de Matiegka } \\
\mathrm{MM}=\text { Peso total }-(\text { peso graso }+ \text { peso óseo }+ \text { peso residual })\end{array}$ \\
\hline 6. & Índice de Masa Corporal & $I M C=\frac{\text { peso }}{\text { estatura }^{2}}$ \\
\hline 7. & Superficie Corporal & $\begin{array}{c}\text { Ecuación de Dubois-Dubois } \\
\mathrm{SC}=0,007184 \times \text { Peso }(\mathrm{kg})^{0,425} \times \text { estatura }(\mathrm{cm})^{0,725}\end{array}$ \\
\hline
\end{tabular}


Tabla IV. Resultados de ecuaciones antropométricas.

\begin{tabular}{|c|c|c|}
\hline 1. & Porcentaje de Grasa Corporal & 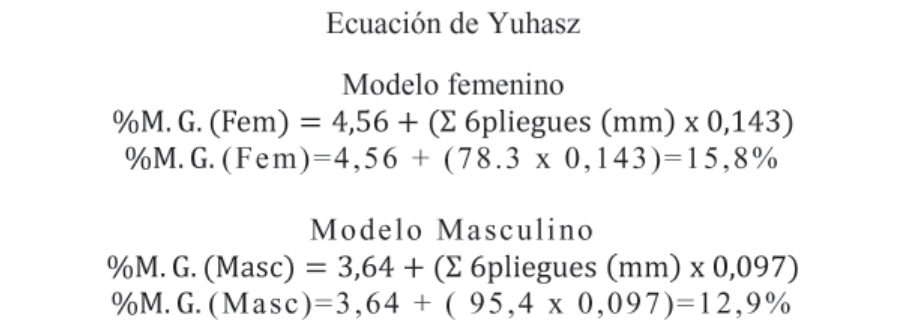 \\
\hline 2. & Masa Grasa & $\begin{array}{c}\text { Peso graso }=\frac{\% \text { de grasa } \times \text { peso total }}{100} \\
\text { Modelo Femenino } \\
\text { Peso graso }(\text { Fem })=\frac{15,8 \times 42}{100}=6,64 \mathrm{~kg} \\
\text { Modelo Masculino } \\
\text { Peso graso }(\text { Masc })=\frac{12,9 \times 76}{100}=9,81 \mathrm{~kg}\end{array}$ \\
\hline 3. & Peso Óseo & $\begin{array}{c}\text { Ecuación de Rocha } \\
\text { ósea Masa }(\mathrm{Kg})=3,02 \times\left(\text { estatura }^{2} \times \mathrm{DM} \times \mathrm{DF} \times 400\right)^{0,712} \\
\text { Modelo femenino } \\
\text { ósea Masa }(\mathrm{Kg})(\mathrm{F})= \\
3,02 \times\left(1,58^{2} \mathrm{~m} \times 50,2 \mathrm{~mm} \times 79,8 \mathrm{~mm} \times 400\right)^{0,712} \\
\text { ósea Masa }(\mathrm{Kg})(\mathrm{F})=3,02 \times(2,50 \mathrm{~m} \times 0,050 \mathrm{~m} \times 0,079 \mathrm{~m} \times 400)^{0,712} \\
\text { ósea Masa }(\mathrm{Kg})(\mathrm{F})=8,03 \mathrm{Kg}\end{array}$ \\
\hline & & $\begin{array}{c}\text { Modelo masculino } \\
\text { ósea Masa }(\mathrm{Kg})(\mathrm{M})= \\
3,02 \times\left(1,72^{2} \mathrm{~m} \times 51,3 \mathrm{~mm} \times 96,7 \mathrm{~mm} \times 400\right)^{0,712} \\
\text { ósea Masa }(\mathrm{Kg})(\mathrm{M})=3,02 \times(3 \mathrm{~m} \times 0,051 \mathrm{~m} \times 0,096 \mathrm{~m} \times 400)^{0,712} \\
\text { ósea Masa }(\mathrm{Kg})(\mathrm{M})=10 \mathrm{Kg}\end{array}$ \\
\hline 4. & Peso Residual & $\begin{array}{c}\text { Ecuación de Würch } \\
\text { Modelo masculino } \\
\text { Masa residual }=(\mathrm{Kg}) \text { Ptx24.1/100(hombres) } \\
\text { Masa residual }=(\mathrm{Kg})(\text { Masc })=\frac{24 \% \times 76 \mathrm{~kg}}{100}=18 \mathrm{Kg} \\
\text { Modelo femenino } \\
\text { Masa residual }=(\mathrm{Kg}) \text { Ptx20.9/ } 100 \text { (mujeres }) \\
\text { Masa residual }=(\mathrm{Kg})(\mathrm{Fem})=\frac{21 \% \times 42 \mathrm{~kg}}{100}=9 \mathrm{Kg}\end{array}$ \\
\hline 5. & Masa Muscular & $\begin{array}{c}\text { Ecuación de Matiegka } \\
\text { MM= Peso total }-(\text { peso graso }+ \text { peso óseo }+ \text { peso residual }) \\
\text { Modelo masculino } \\
\mathrm{MM}(\text { Mas })=76 \mathrm{Kg}-(9,81 \mathrm{Kg}+10 \mathrm{Kg}+18 \mathrm{Kg})=38 \mathrm{Kg} \\
\text { Modelo femenino } \\
\mathrm{MM}(\text { Fem })=42 \mathrm{Kg}-(6,64 \mathrm{Kg}+8,03 \mathrm{Kg}+9 \mathrm{Kg})=18 \mathrm{Kg}\end{array}$ \\
\hline
\end{tabular}


Tabla IV. Resultados de ecuaciones antropométricas.

\begin{tabular}{|c|c|c|}
\hline 6. & Índice de Masa Corporal & $\begin{array}{l}\qquad \text { IMC }=\frac{\text { peso }}{\text { estatura }^{2}} \\
\text { Modelo masculino } \\
\\
\text { Modelo femenino } \\
\text { IMC (Mas) }=\frac{76}{1,72^{2}}=26 \\
\\
\end{array}$ \\
\hline 7. & Superficie Corporal & $\begin{array}{c}\text { Dubois-Dubois } \\
\mathrm{SC}=0,007184 \times \text { Peso }(\mathrm{kg})^{0,425} \times \text { estatura }(\mathrm{cm})^{0,725} \\
\text { Modelo femenino } \\
\mathrm{SC}(\text { Fem })=0,007184 \times 42(\mathrm{~kg})^{0,425} \times 158(\mathrm{~cm})^{0,725} \\
\mathrm{SC}(\text { Fem })=0,007184 \times 4,90 \times 39,2=1,38 \mathrm{~m}^{2} \\
\text { Modelo masculino } \\
\text { SC (Masc. })=0,007184 \times 76(\mathrm{~kg})^{0,425} \times 172(\mathrm{~cm})^{0,725} \\
\text { SC (Masc. })=0,007184 \times 6,3 \times 41.7=1,84 \mathrm{~m}^{2}\end{array}$ \\
\hline
\end{tabular}

Y respecto del índice de la masa corporal, el resultado para la mujer fue de 17 , que de acuerdo a la tabla de la OMS de 1995 , se interpreta como bajo peso y la interpretación para el hombre es 26 , se interpreta como sobrepeso y finalmente la superficie corporal para la mujer fue de $1.38 \mathrm{~m} 2 \mathrm{y}$ para el hombre $1.84 \mathrm{~m} 2$.

\section{CONCLUSIONES}

Es posible usar ecuaciones antropométricas específicas según la edad y sexo para calcular los compartimentos corporales a través de las mediciones que se seleccionaron. Las ecuaciones generadas en este estudio se basaron en una técnica práctica, sencilla, de bajo costo y adecuada para calcular la composición corporal. En cuanto a los diámetros del esqueleto proporcionan información de la cantidad de la masa ósea. El hecho que estas ecuaciones estén validadas por los autores que la publican no significa que sea válida para otra población.

El uso de estas ecuaciones de Yuhasz, Rocha, Würch y Matiegka, para calcular la composición corporal puede ser viable para su aplicación en poblaciones adultas. Se requiere hacer más estudios con una muestra más grande para verificar si estas son representativas y puedan tener una correlación entre las medidas, las ecuaciones y los grupos para Colombia.

\section{REFERENCIAS}

[1] Z. M. Wang, R.N. Pierson, JR y S.B. Heymsfield. The five-level model: a new approach to organizing body composition research. Am. J. Clin. Nutr. 56: 19-28. 1992.

[2] M. D. Marrodán, M. González, C. Prado. Antropología de la Nutrición. Métodos, Técnicas y Aplicaciones. Ed. Noesis. Madrid, 1995.

[3] R. Casanova. Técnicas de valoración del estado nutricional. Vox Paediatrica. 11 (1), 26-35. España. 2003.

[4] J. Sirvent \& R. Garrido. Valoración antropométrica de la composición corporal. Universidad de Alicante. España. 2009.

[5] M. Gibney, H. Vorster, \& F. Kok. Introducción a la nutrición humana. España. 2005.

[6] A. R. Frisancho. Anthropometric Standards for the Assessment of Growth and Nutritional Status. The University of Michigan Press. Michigan. 1990. 
[7] A. Quetelet. Fisica Sociale ossia svolgimento delle facoltá dell' uomo Cap. 2: Relazioni tra il peso e la statura. In: Economía Politica, G. Boccardo (ed.), Torino: Unione Tipografico-Editrice Torinese, 1875.

[8] V. Moreno, J. Gómez, M. Antoranz. Medición de la grasa corporal mediante impedancia Bioeléctrica, pliegues cutáneos y ecuaciones a partir de Medidas antropométricas. Análisis comparativo. Centro de Salud Coronel de Palma. Móstoles. Madrid. Rev Esp Salud Pública 2001; 75: 221-236. 2001.

[9] M.Sillero. Composición corporal. Facultad de ciencias de la actividad física y del deporte (I.N.E.F). Universidad Politécnica de Madrid. 2005.

[10] Malagón, C. (2001). Manual de antropometría. Banco de la República. Bogotá.

[11] G. Arechavaleta, H. Castillo, H. Herrera \& M. Pacheco. Composición Corporal en una población de estudiantes universitarios. Revista de la Facultad de Medicina, 25, 209-216. 2002.

[12] M. S. L. Rocha. Peso ósseo do brasileiro de ambos os sexos de 17 a 25 años. Arquivos de Anatomia e Antropologia, 1, 445-451. 1975.

[13] J. Alvero. Protocolo de valoración de la composición corporal para el reconocimiento médico-deportivo, documento de consenso del grupo español de cineantropometría de la federación Española de medicina y deporte, Disponible en: http:// femede.es/documentos/ConsensoCine 131.pdf. Volumen XXVI. Número 131. Págs.166-179. 2009.

[14] C. Rosen. Osteoporosis. In: Goldman L, Schafer AI, eds. Goldman's Cecil Medicine. 24th ed. Philadelphia, PA: Elsevier Saunders; chap 25. 2012.

[15] J. Fernandez, R. Aguilera. Estimación de la masa muscular por diferentes ecuaciones antropométricas en levantadores de pesas de alto nivel. Archivos de medicina y deporte. 2001.

[16] R. Garrido, M. González, I. Coll. Comparación de las fórmulas de Lee y Martin para el cálculo de la masa muscular de 3125 deportistas de alto nivel. Servicios de Apoyo al Deportista del Centro de Tecnificación de Alicante (España) 2005.

[17] J. C. Aristizábal, M. T. Restrepo, A. Estrada. Evaluación de la composición corporal de adultos sanos por antropometría e impedancia bioeléctrica. Grupo de Investigación en Alimentación y Nutrición Humana, Escuela de Nutrición y Dietética, Universidad de Antioquia, Medellín, Colombia Biomédica 2007.

[18] P. Pacheco. Ambidiestrismo y el pensamiento visual. Más allá del dibujo. Centro de Publicaciones de la Universidad Nacional de Colombia sede en Manizales, 1996. 